\title{
Enhanced operational stability through interfacial modification by active encapsulation of perovskite solar cells
}

Cite as: Appl. Phys. Lett. 116, 113502 (2020); https://doi.org/10.1063/1.5144038

Submitted: 05 January 2020 . Accepted: 29 February 2020 . Published Online: 19 March 2020

Sudeshna Chosh, Roja Singh, Anand S. Subbiah, Pablo P. Boix (D), Iván Mora Seró, and Shaibal K. Sarkar (iD)

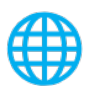

Lock-in Amplifiers Find out more today

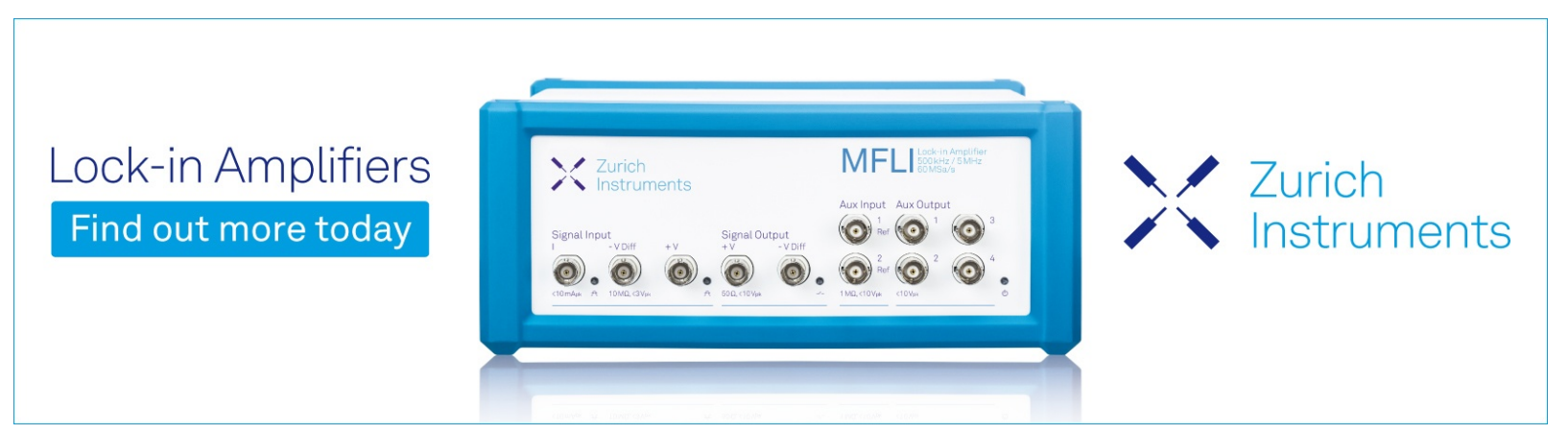

Zurich

Instruments 


\title{
Enhanced operational stability through interfacial modification by active encapsulation of perovskite solar cells
}

Cite as: Appl. Phys. Lett. 116, 113502 (2020); doi: 10.1063/1.5144038

Submitted: 5 January 2020 - Accepted: 29 February 2020 •

Published Online: 19 March 2020

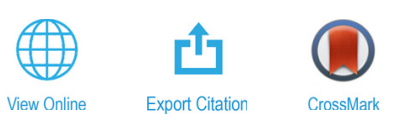

Sudeshna Ghosh, ${ }^{1}$ Roja Singh, ${ }^{2}$ Anand S. Subbiah, ${ }^{2}$ Pablo P. Boix, ${ }^{3}$

Iván Mora Seró, ${ }^{4}$ and Shaibal K. Sarkar ${ }^{2, a)}$ (D)

\begin{abstract}
AFFILIATIONS
${ }^{7}$ Center of Research in Nanotechnology and Science, Powai, Mumbai 400 076, India

${ }^{2}$ Department of Energy Science and Engineering, Indian Institute of Technology Bombay, Powai, Mumbai 400 076, India

${ }^{3}$ Institut de Ciència dels Materials, University of Valencia Catedràtic J. Beltran 246980 Paterna, Valencia, Spain

${ }^{4}$ Institute of Advanced Materials, Universitat Jaume I, Av. de Vicent Sos Baynat, s/n 12071Castelló de la Plana, Spain
\end{abstract}

${ }^{a)}$ Author to whom correspondence should be addressed: shaibal.sarkar@iitb.ac.in

\begin{abstract}
Encapsulates are, in general, the passive components of any photovoltaic device that provides the required shielding from the externally stimulated degradation. Here we provide comprehensive physical insight depicting a rather non-trivial active nature, in contrast to the supposedly passive, atomic layer deposition (ALD) grown $\mathrm{Al}_{2} \mathrm{O}_{3}$ encapsulate layer on the hybrid perovskite $\left[\left(\mathrm{FA}_{0.83} \mathrm{MA}_{0.17}\right)_{0.95} \mathrm{Cs}_{0.05} \mathrm{PbI}_{2.5} \mathrm{Br}_{0.5}\right]$ photovoltaic device having the configuration: glass/FTO/SnO $2 /$ perovskite/spiro-OMeTAD/Au/ $( \pm) \mathrm{Al}_{2} \mathrm{O}_{3}$. By combining various electrical characterization techniques, our experimental observations indicate that the ALD chemistry produces considerable enhancement of the electronic conductivity of the spiro-OMeTAD hole transport medium (HTM), resulting in electronic modification of the perovskite/HTM interface. Subsequently, the modified interface provides better hole extraction and lesser ionic accumulation at the interface, resulting in a significant lowering of the burn-in decay and nearly unchanged charge transport parameters explicitly under the course of continuous operation. Unlike the unencapsulated device, the modified electronic structure in the $\mathrm{Al}_{2} \mathrm{O}_{3}$ coated device is essentially the principal reason for better performance stability. Data presented in this communication suggest that the ionic accumulation at the spiro-OMeTAD/perovskite interface triggers the device degradation in the uncoated devices, which is eventually followed by material degradation, which can be avoided by active encapsulation.
\end{abstract}

Published under license by AIP Publishing. https://doi.org/10.1063/1.5144038

Intrinsic $^{1,2}$ and extrinsic ${ }^{3-6}$ routes of degradation are the primary bottleneck that thwart the commercial realization of hybrid perovskite solar cells (PSC). Prevailing measures taken to avoid extrinsic degradation $^{7,8}$ are efficient encapsulates to protect the active device, mainly from humidity and oxygen.

In this context, recent reports ${ }^{9-11}$ suggest the applicability of an atomic layer deposition (ALD) grown $\mathrm{Al}_{2} \mathrm{O}_{3}$ layer as a preventive measure against the degradation, leading to improved device performance under diurnal cycles. ${ }^{12}$ The surface-limited ingrowth of the as-deposited $\mathrm{Al}_{2} \mathrm{O}_{3}$ in the spiro-OMeTAD layer during the nucleation period essentially holds the key, which makes this technique an efficient measure for the long-term stability of the device. ${ }^{13}$

In addition to the conventional extrinsic degradation mechanisms, recent studies report factors that can accelerate the degradation process under certain measurement conditions, increasing the induced stress. ${ }^{14}$ Factors like duration, periodicity of measurements, ${ }^{15}$ and preconditioning conditions ${ }^{16}$ also play an important role in the overall device stability. Generally, induced degradation is often (partially) recoverable $\mathrm{e}^{17}$ and is majorly attributed to the inappropriate distribution of the activated ions. ${ }^{18}$ However, under continuous operation, these damages collectively initiate a high level of severity resulting in irreversible degradation. Thus, one could find radical differences in device stability when comparing the performance under intermittent and continuous measurement conditions. ${ }^{19}$ When the device is under a relaxing condition in the dark, the redistribution of the mobile ions rejuvenates the device. ${ }^{20}$ However, under uninterrupted measurement, efficient charge extraction in conjunction with ideal band alignment encourages the device stability. ${ }^{21,22}$ Thus, in the absence of external entities, proper band alignment and chemical inactivity of the ionic species with 
the charge transport layers are some of the vital issues to ensure the enhanced device stability even under a stressed condition.

We have recently shown ${ }^{23}$ that $\mathrm{ALD}$-grown $\mathrm{Al}_{2} \mathrm{O}_{3}$ on top of the device escalates the shelf life of the device and enhances the device life under continuous illumination. Although the increased shelf life indicates impermeability against ambient constituents, it is interesting to observe favorable modifications of the interfacial characteristics under continuous illumination. In this communication, we unveil the unexpected modifications in the perovskite/spiro-OMeTAD interfacial electronic characteristic upon $\mathrm{Al}_{2} \mathrm{O}_{3}$ coating on top of the device. The conductivity of the HTM improves upon $\mathrm{Al}_{2} \mathrm{O}_{3}$ ingrowth, a counterintuitive phenomenon with a beneficial effect in the charge extraction interface. As a result, the "ALD-encapsulation" demotes charge and ion accumulation at the interface, suppressing chemical modification under continuous operation substantially in comparison to pristine uncoated devices.

Herein, we fabricate PSCs using the extended multi-cation and multi-anion perovskite $\left[\left(\mathrm{FA}_{0.83} \mathrm{MA}_{0.17}\right)_{0.95} \mathrm{Cs}_{0.05} \mathrm{PbI}_{2.5} \mathrm{Br}_{0.5}\right]$ as an active layer with bilayer $\mathrm{SnO}_{2}$ and $\mathrm{Li}$-doped spiro-OMeTAD as electron transport layer (ETL) and hole transporting layer (HTL), respectively, as shown in Fig. S1 in the supplementary material. ALD-grown $\mathrm{Al}_{2} \mathrm{O}_{3}$ is deposited on top of the processed device at $75^{\circ} \mathrm{C}$ using trimethyl aluminum and water inside a customized flow reactor. The details of the device fabrication and the ALD process parameters are described in the supplementary material (experimental section).

A passive encapsulation effect should be expected from the $\mathrm{Al}_{2} \mathrm{O}_{3}$ coating, but surprisingly we report here that the $\mathrm{Al}_{2} \mathrm{O}_{3}$ encapsulation process also had an active impact on the device's electronic characteristics. Apart from the previously reported long-term stability, ${ }^{23}$ the major differences between the pristine and coated devices during the burn-in period essentially justify the improved light induced degradation of the coated sample over the pristine ones as shown in Figs. 1(a) and $1(\mathrm{~b})$. The burn-in decay is most prominent under the shortcircuit condition in comparison with open-circuit; hence, we mostly concentrate our discussion here toward short-circuit conditions ( $\mathrm{J}_{\mathrm{SC}}$ ). However, for comparison, the measurements under open circuit conditions $\left(\mathrm{V}_{\mathrm{OC}}\right)$ are also depicted in Fig. $\mathrm{S} 2$ in the supplementary
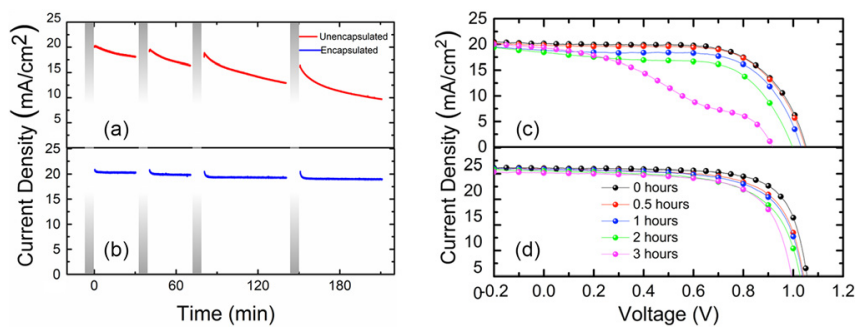

FIG. 1. $J_{S C}$ vs time for (a) unencapsulated and (b) encapsulated devices along with intermittent J-V (forward direction) for (c) unencapsulated and (d) encapsulated devices measured at 0,30 min, $60 \mathrm{~min}, 120 \mathrm{~min}$, and $180 \mathrm{~min}$. The gray section represents the time regime in which some other experiments are done as described in the experimental section. In Fig. 1(a), a sharp decrease in the current density is observed with time in unencapsulated devices, whereas in (b) no reduction in current density is visible in encapsulated devices. Along with $J_{S C}$ vs time, intermittent light J-V of unencapsulated devices shows a visible decrement of FF resulting in a kink (S-shape) formation. On the flipside, no such decrement is observed in intermittent $\mathrm{J}-\mathrm{V}$ of encapsulated devices. material. It is also worth mentioning that under intermittent measurements where the device undergoes relaxation between successive measurements, there is little difference in the photovoltaic device parameters between these competitive devices as shown in Fig. S3 in the supplementary material. ${ }^{24}$ To better understand the changes of electronic nature that the device undergoes during the burn-in period, we constantly measured the $\mathrm{J}_{\mathrm{SC}}$ for the first three hours while intermittently recorded the $\mathrm{J}-\mathrm{V}$ sweep (both forward/reverse direction and under light/dark condition) along with electrochemical impedance spectroscopy (EIS) measurements. Experimental details on the intermittent measurement procedure are further briefed in the supplementary material.

The shorter time interval in which the devices underwent considerable loss in efficiency is of specific interest, past that the coated device retained a stable performance for up to $100 \mathrm{~h}$ while the pristine devices continue degrading. ${ }^{23}$ The evolution of $\mathrm{J}_{\mathrm{SC}}$ with time is depicted in Figs. 1(a) and 1(b) for pristine and encapsulated devices, respectively. The pristine devices exhibit a sharp decline in the shortcircuit current $\left(\mathrm{J}_{\mathrm{SC}}\right)$ over time, where the $\mathrm{J}_{\mathrm{SC}}$ dropped to $48 \%$ of its initial value $\left(20 \mathrm{~mA} / \mathrm{cm}^{2}-9.70 \mathrm{~mA} / \mathrm{cm}^{2}\right)$ within a span of $3 \mathrm{~h}(180 \mathrm{~min})$. In contrast, the encapsulated cells retained almost $92 \%$ of the initial value by the end of $3 \mathrm{~h}(180 \mathrm{~min})\left(20.7 \mathrm{~mA} / \mathrm{cm}^{2}-19 \mathrm{~mA} / \mathrm{cm}^{2}\right) . \mathrm{J}-\mathrm{V}$ characteristics of the pristine and encapsulated device are measured intermittently to follow the individual device parameters during the continuous $\mathrm{J}_{\mathrm{SC}}$ measurements, as shown in Figs. 1(c) and 1(d). Although we observed slight changes in $\mathrm{V}_{\mathrm{OC}}$ and specifically in $\mathrm{FF}$ from the intermittent $\mathrm{J}-\mathrm{V}$ characteristics of the encapsulated device, the pristine unencapsulated devices, in contrast, display a significant degradation of the $\mathrm{J}-\mathrm{V}$ characteristics.

Beyond the decrease in the $\mathrm{J}_{\mathrm{SC}}$ and $\mathrm{V}_{\mathrm{OC}}$, a significant reduction in the fill-factor is clearly observed, as parametrically depicted in Fig. S4 in the supplementary material. The striking difference is the unprecedented appearance of the S-shape kink (after $3 \mathrm{~h}$ (180 min) here, but typically varies with the sample) in the pristine devices, see Fig. 1(d), which is not observed in the encapsulated devices. Such a peculiar device characteristic in these pristine devices indicates inefficient charge extraction processes, possibly due to the emergence of the energy barrier ${ }^{25}$ or a disadvantageous effect due to the time dependent modification of the internal electric field. ${ }^{26}$ Both scenarios are essentially a result of the charge accumulation across the interface, prohibiting the movement of charge carriers from perovskite to the HTL. Because in both the competitive devices, the ETL $\left(\mathrm{SnO}_{2}\right)$ or ETL/ perovskite interface has the least possibility of modification due to the implementation of $\mathrm{Al}_{2} \mathrm{O}_{3}$ encapsulation, it is logical to ignore any probabilistic effect that may arise from this interface. Upon dark recovery $(\sim 72 \mathrm{~h})$, the device recovers its normal trend and the salient S-type feature disappears, as shown in Fig. S5 in the supplementary material, which results in a partially reversible phenomenon. As previously reported, keeping the measured cells under dark conditions allows the redistribution of ions. This paves the understanding that the appearance of the kink in the J-V characteristic is probably due to the non-uniform spatial redistribution of the photo-activated mobile ions that re-appropriates upon relaxation. The temporal modification of the built-in electric field due to ionic accumulation across the interfaces, activated by bias and light, is also evident from the inversion in hysteric effects, ${ }^{27}$ which is observed alongside the S-type features. The current-voltage hysteresis usually described in the literature is 
characterized by higher performance in reverse scan (RS) compared to the forward scan (FS). In Figs. S6(a) and S6(b) in the supplementary material, we can see the inversion in hysteresis in the J-V curve by the end of $3 \mathrm{~h}$ (180 min), which gets reversed upon relaxation under dark. This concurrently suggests the modification of the built-in electric field in the device due to the incongruous spatial distribution of the photoactivated ions, alongside inappropriate band-alignment. While the inversion in hysteresis in unencapsulated devices is evident, the intensity of the phenomenon is almost invariant in the encapsulated cells along with a lesser hysteresis index in the encapsulated devices compared to unencapsulated ones as shown in Fig. S6(c) in the supplementary material.

The adverse effects of mobile ions in the pristine devices are evident even in the dark J-V measurements performed intermittently following the postillumination measurements. The crossover voltage (where, J is minimum) between the forward and the reverse scans, as shown in Fig. 2 and the hysteresis Figs. S7(a)-S7(e) in the supplementary material for the pristine devices, are found recurrently increasing with time while such changes are essentially absent in the encapsulated devices.

Open circuit voltage decay (OCVD) can be useful to understand the active behavior of the ALD layer as it provides information on the internal energetics of the devices. These measurements were performed on devices with the same structure under different light soaking conditions $(2,6$, and $10 \mathrm{~min})$. Here, the light soaking time is deliberately reduced compared to the previous experiments, avoiding any degradation through an extrinsic parameter, hence reflecting the effect of the photo-stimulated ions. Our results invariably indicate that the relaxation time constant $\left(\tau_{\text {rec }}\right)^{28}$ strictly depends on the illumination time for the pristine devices as shown Figs. S8(a) and S8(b) in the supplementary material. In contrast, the OCVD and, hence, the $\tau_{\text {rec }}$ value remain invariant for the encapsulated devices as shown in

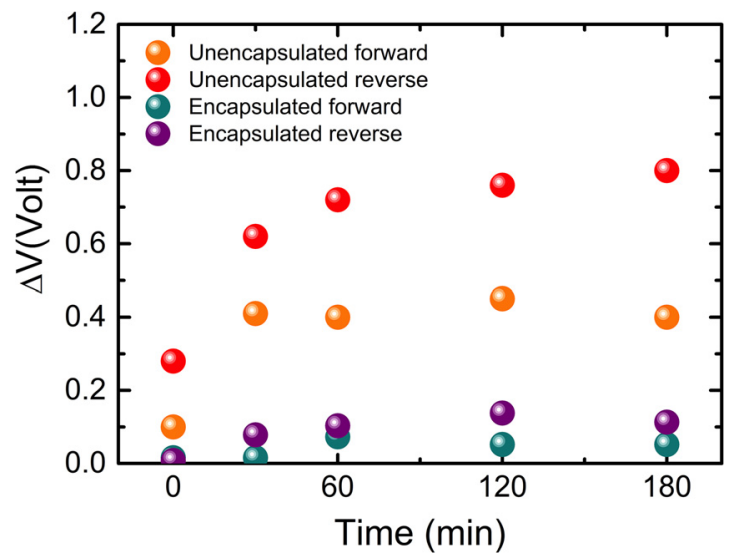

FIG. 2. Variation of hysteretic voltage $(\Delta \mathrm{V})$ calculated from dark $\mathrm{J}-\mathrm{V}$ measurements (shown in Fig. S7), and the position of crossover points of unencapsulated and encapsulated devices for both the forward and reverse direction with time for $0 \mathrm{~min}, 30 \mathrm{~min}, 60 \mathrm{~min}, 120 \mathrm{~min}$, and $180 \mathrm{~min}$. In unencapsulated devices, the crossover voltage value increases with time which means some slow phenomena contribute to this offset. In encapsulated devices, the voltage where current is changing the direction is almost constant in both directions with measurement time. Slow phenomena that contribute to the offset are essentially absent in encapsulated devices.
Figs. S8(c) and S8(d) in the supplementary material (and explanation thereon). Also, the net OCVD is faster in the pristine sample than in the encapsulated ones that have a sustaining electrostatic potential for a considerably longer timescale as shown in Figs. S8(a) and S8(b). Considering the time span of the experiments, the OCVD measurements have little to do with the degradations of the cells; rather, they indicate the effect is also clearly evident in the low frequency capacitance measurements. In this line, frequency dependent capacitance measurements under dark portray a significant change in the electrode polarization $^{29-31}$ as shown in Fig. S9 in the supplementary material. Prior to any illumination exposure, our measurements reveal a capacitance value of $7.14 \times 10^{-6} \mathrm{~F} / \mathrm{cm}^{2}$ at $0.1 \mathrm{~Hz}$ for unencapsulated devices, an order of magnitude higher that the encapsulated one, as shown in Fig. S9 in the supplementary material. However, the transition frequency $\left(f_{s}\right)$ remains the same in both the devices $\left(\mathrm{ca} .10^{1}-10^{2} \mathrm{~Hz}\right)$ with similar dielectric polarization, while the electrode polarization gets reduced upon $\mathrm{Al}_{2} \mathrm{O}_{3}$ coating, indicating the better interfacial quality of encapsulated devices. Figure $\mathrm{S10}(\mathrm{a})$ in the supplementary material shows a non-capacitive current under slow-scan rate, indicating the impact of chemical modification of the perovskite-HTM interface, specifically in the pristine devices. Such irreversible non-capacitive effect $^{32,33}$ that may originate the permanent degradation of the pristine devices is distinctly absent in the coated devices as shown in Fig. S10(b) in the supplementary material, probably due to the favorable band alignment at the interface.

We further performed the electrochemical impedance spectroscopy (EIS), under illumination, to gain insight into the underlying mechanisms of these effects. Generally, the ETL/perovskite interface has a significant influence on the parameters depicted in EIS, partly because the mobility of electrons is much higher. ${ }^{34,35}$ It is evident through the literature that in a given system, the charge separation and extraction process is not only influenced by the ETL/perovskite interface but also the perovskite/HTL interface. Here, EIS has measured under AM 1.5 G illumination at open circuit conditions, intermittently along with $\mathrm{J}-\mathrm{V}$ and results in the characteristic pattern for perovskite solar cells as shown in Fig. 3.

The impedance spectra are analyzed with an equivalent circuit which consists of a series resistance $\left(\mathrm{R}_{\mathrm{s}}\right)$ accounting for the purely resistive effects of cables, transparent conducting oxide (TCO), etc., a resistor where recombination and charge transport/transfer effects are coupled $\left(\mathrm{R}_{\mathrm{rec}+\mathrm{tr}}\right)$, the geometric capacitance of the device $\left(\mathrm{C}_{\mathrm{g}}\right)$, and a
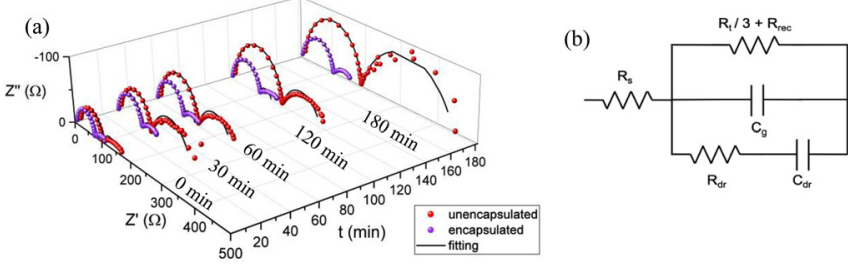

FIG. 3. (a) Nyquist plot measured in light at open circuit condition for unencapsulated (red) and encapsulated (purple) for $0 \mathrm{~min}, 30 \mathrm{~min}, 60 \mathrm{~min}, 120 \mathrm{~min}$, and $180 \mathrm{~min}$ and $(\mathrm{b})$ the equivalent circuit including $\mathrm{Cg}$ is geometrical capacitance, a combination of dielectric relaxation and transport resistance $R_{t}$ and recombination resistance $R_{\text {rec. }}$. The size of two arcs changes with time in unencapsulated devices as the measurement time progresses, whereas the size remains almost unchanged in encapsulated devices. 
parallel branch, including a capacitor $\left(\mathrm{C}_{\mathrm{dr}}\right)$ and a resistor $\left(\mathrm{R}_{\mathrm{dr}}\right)$ in series. ${ }^{36}$ While the rest of the parameters do not show significant changes in time, as shown in Fig. S11 in the supplementary material, $\mathrm{R}_{\mathrm{rec}+\mathrm{tr}}$ displays a clear increase, see Fig. 4. In particular, $\mathrm{R}_{\mathrm{rec}+\mathrm{tr}}$ increases more abruptly in the unencapsulated case. It is worth to remark that this parameter is obtained from the total resistance of both arcs in the Nyquist plots. It contains the coupled contributions of recombination and a third of charge transport, including that of the selective contacts and interfaces. Although the combinations of different effects in the same resistance hinder the numeric interpretation of the individual components, the feedback obtained from the J-V enables a qualitative characterization of the degradation mechanisms. In our case, the initial effect of the encapsulation is reflected in a slight decrease in the $R_{\text {rec }+ \text { tr }}$, which is attributed to the increase in the hole selective material conductivity as shown in Fig. S12 in the supplementary material which is well supported by other experimental shreds of evidence. Figure S12 in the supplementary material shows an order of increase in the lateral conductivity of the spiro-OMeTAD upon $\mathrm{Al}_{2} \mathrm{O}_{3}$ coating. Considering the evolution of the $\mathrm{V}_{\mathrm{OC}}$, one would expect the decrease in the recombination resistance to be more intense in the unencapsulated case. Nonetheless, $\mathrm{R}_{\mathrm{rec}+\text { tr }}$ increases with time, which reflects a deterioration of the charge transport characteristics of the device. As evident from FF evolution as well, the encapsulation strongly slows down this process. In this sense, the transport properties deterioration, considering other supporting experimental observations as discussed previously, indicates an increase in the charge transfer resistance at the interface.

Our conclusion is in accord with the similar hypothesis put forwarded by Schulz et al., ${ }^{37}$ who rightfully indicated that the offset between the perovskite-spiro-OMeTAD plays an important role in the charge extraction efficiency of that interface. An improper offset that gives rise to inefficient charge extraction has a detrimental effect on the device performance. Improved conductivity in the HTM upon ALD alumina deposition essentially implies the increased carrier concentration in the material; hence, the electronic band structure gets modified. Figure S13 in the supplementary material shows an increment of the work function $0.15-0.2 \mathrm{eV}$ upon $\mathrm{Al}_{2} \mathrm{O}_{3}$ coating from

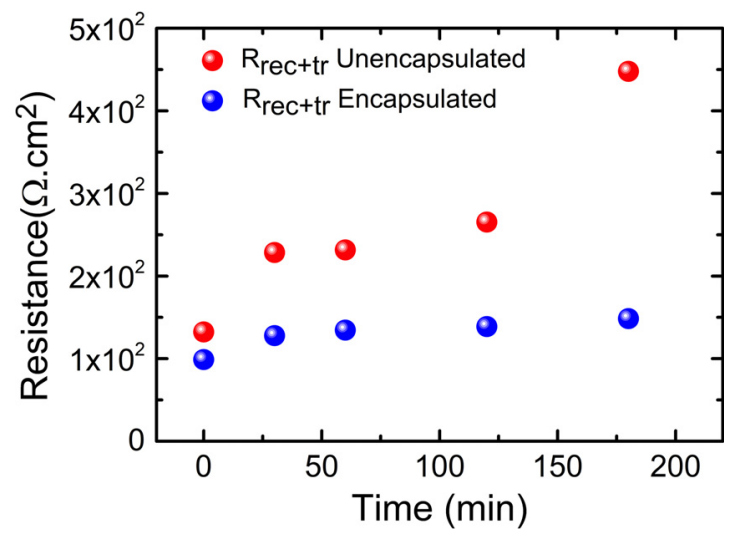

FIG. 4. Variation of combined effect of recombination resistance and charge transfer resistance for unencapsulated (red) and encapsulated (blue) devices with time $0 \mathrm{~min}, 30 \mathrm{~min}, 60 \mathrm{~min}, 120 \mathrm{~min}$, and180 min. The value of $R_{\text {rec }+ \text { tr }}$ of unencapsulated devices increases with time but it is almost constant in encapsulated devices. ultraviolet photoelectron spectroscopy (UPS), indicating probabilistic changes in the interfacial (perovskite/spiro-OMeTAD) band structure. The modified interfacial electronic band structure upon $\mathrm{Al}_{2} \mathrm{O}_{3}$ coating discourages ion-induced modifications of the interfaces in encapsulated devices. In contrast, for the pristine devices, ion-induced modification of the interface is evident, resulting in inefficient charge extraction and charge carrier accumulation across the perovskite/ spiro-OMeTAD interfaces. This ion-induced detrimental modification becomes more severe in the slow scan rate where long polarization happens, causing chemical degradation, known as the non-capacitive effect. $^{38}$ Again the inverted hysteresis, as shown in Fig. S6, is also a result of the unfavorable band bending. It was suggested that the accumulated ions under reverse scan might enhance the internal drift field in bulk but generated reversed electric field regions at the interfaces. The inversion of the hysteresis indeed is a fingerprint of the development of the inefficient extraction barrier due to the change in the local electric field derived from the inappropriate distribution of the mobile ions.

In summary, our experimental observations lead to the conclusion that in the presence of the preferred extraction barrier, interfacial ion-accumulation is prohibited in $\mathrm{Al}_{2} \mathrm{O}_{3}$ coated devices resulting in better intrinsic stability. Significantly, $\mathrm{Al}_{2} \mathrm{O}_{3}$ produces an active effect in the perovskite layer, affecting its behavior beyond the simple passive role supposed for an encapsulation system. Improved conductivity of the spiro-OMeTAD upon $\mathrm{Al}_{2} \mathrm{O}_{3}$ coating also influences the perovskite-HTL interfacial band structure. Lowering of capacitance in the electrode polarization regime with negligible non-capacitive current and above all the absence of the s-type kink in the J-V characteristics unequivocally proves the proposed hypothesis. In contrast, for pristine devices the inefficient charge extraction, probably due to temporally varying interfacial electronic-band structure, can be the primal cause that paves the way to the degradation of the devices. Based on our understanding, we suggest that interface degradation takes place before the material degradation is initiated. These results pave the way for the development of encapsulation systems that act synergistically on both the active layer performance and the long term stability beyond conventional encapsulation that merely acts as a blocking system, with no direct effect of the working behavior of the active layer.

See the supplementary material for full discussion of the experimental section.

The authors acknowledge the financial support from the Ministry of New and Renewable Energy, Government of India. S.G. thanks the University Grant Commission, Government of India for financial support. R.S. thanks the German Academic Exchange Service (DAAD) for financial support. Authors thank Neha Mahuli and Bireswar Mandol for their timely help with ALD. P.P.B. would like to thank the Ministerio de Economíay Competitividad of Spain for the funding through the Project No. MAT2017-88905-P and his RyC contract; and Generalitat Valenciana (No. SEJI2017/2017/012).

\section{REFERENCES}

${ }^{1}$ J. A. McLeod and L. Liu, J. Phys. Chem. Lett. 9, 2411 (2018).

${ }^{2}$ P. Yadav, D. Prochowicz, E. A. Alharbi, S. M. Zakeeruddin, and M. Grätzel, J. Mater. Chem. C 5, 7799 (2017). 
${ }^{3}$ N.-K. Kim, Y. H. Min, S. Noh, E. Cho, G. Jeong, M. Joo, S.-W. Ahn, J. S. Lee, S. Kim, K. Ihm, H. Ahn, Y. Kang, H.-S. Lee, and D. Kim, Sci. Rep. 7, 4645 (2017). ${ }^{4} \mathrm{M}$. Salado, L. Contreras-Bernal, L. Caliò, A. Todinova, C. López-Santos, S. Ahmad, A. Borras, J. Idígoras, and J. A. Anta, J. Mater. Chem. A 5, 10917 (2017).

${ }^{5}$ H. J. Jung, D. Kim, S. Kim, J. Park, V. P. Dravid, and B. Shin, Adv. Mater. 30, 1802769 (2018)

${ }^{6}$ N. Aristidou, C. Eames, I. Sanchez-Molina, X. Bu, J. Kosco, M. Saiful Islam, and S. A. Haque, Nat. Commun. 8, 15218 (2017).

${ }^{7}$ R. Cheacharoen, N. Rolston, D. Harwood, K. A. Bush, R. H. Dauskardt, and M. D. McGehee, Energy Environ. Sci. 11, 144 (2018).

${ }^{8}$ L. Shi, T. L. Young, J. Kim, Y. Sheng, L. Wang, Y. Chen, Z. Feng, M. J. Keevers, X. Hao, P. J. Verlinden, M. A. Green, and A. W. Y. Ho-Baillie, ACS Appl. Mater. Interfaces 9, 25073 (2017).

${ }^{9}$ X. Dong, X. Fang, M. Lv, B. Lin, S. Zhang, J. Ding, and N. Yuan, J. Mater. Chem. A 3, 5360 (2015)

${ }^{10}$ S. Seo, S. Jeong, C. Bae, N.-G. Park, and H. Shin, Adv. Mater. 30, 1801010 (2018).

${ }^{11}$ D. Koushik, W. J. H. Verhees, Y. Kuang, S. Veenstra, D. Zhang, M. A. Verheijen, M. Creatore, and R. E. I. Schropp, Energy Environ. Sci. 10, 91 (2017).

${ }^{12}$ M. V. Khenkin, K. M. Anoop, I. Visoly-Fisher, S. Kolusheva, Y. Galagan, F. Di Giacomo, O. Vukovic, B. R. Patil, G. Sherafatipour, V. Turkovic, H.-G. Rubahn, M. Madsen, A. V. Mazanik, and E. A. Katz, ACS Appl. Energy Mater. 1, 799 (2018).

${ }^{13}$ C. A. Wilson, R. K. Grubbs, and S. M. George, Chem. Mater. 17, 5625 (2005).

${ }^{14}$ W. Luo, Y. S. Khoo, P. Hacke, V. Naumann, D. Lausch, S. P. Harvey, J. P. Singh, J. Chai, Y. Wang, A. G. Aberle, and S. Ramakrishna, Energy Environ. Sci. 10, 43 (2017).

${ }^{15}$ L. Jiang, J. Lu, S. R. Raga, J. Sun, X. Lin, W. Huang, F. Huang, U. Bach, and Y.B. Cheng, Nano Energy 58, 687 (2019).

${ }^{16}$ M. De Bastiani, G. Dell'Erba, M. Gandini, V. D’Innocenzo, S. Neutzner, A. R. S. Kandada, G. Grancini, M. Binda, M. Prato, J. M. Ball, M. Caironi, and A. Petrozza, Adv. Energy Mater. 6, 1501453 (2016).

${ }^{17}$ J. Carolus, T. Merckx, Z. Purohit, B. Tripathi, H.-G. Boyen, T. Aernouts, W. De Ceuninck, B. Conings, and M. Daenen, Sol. RRL 3, 1900226 (2019).

${ }^{18}$ M. V. Khenkin, K. M. Anoop, E. A. Katz, and I. Visoly-Fisher, Energy Environ. Sci. 12, 550 (2019).

${ }^{19}$ M. V. Khenkin, K. M. Anoop, I. Visoly-Fisher, Y. Galagan, F. Di Giacomo, B. R. Patil, G. Sherafatipour, V. Turkovic, H.-G. Rubahn, M. Madsen, T. Merckx,
G. Uytterhoeven, J. P. A. Bastos, T. Aernouts, F. Brunetti, M. Lira-Cantu, and E. A. Katz, Energy Environ. Sci. 11, 739 (2018).

${ }^{20}$ R. Gottesman, P. Lopez-Varo, L. Gouda, J. A. Jimenez-Tejada, J. Hu, S. Tirosh, A. Zaban, and J. Bisquert, Chem 1, 776 (2016).

${ }^{21}$ D. Shin, D. Kang, J. Jeong, S. Park, M. Kim, H. Lee, and Y. Yi, J. Phys. Chem. Lett. 8, 5423 (2017).

${ }^{22}$ C. Ding, Y. Zhang, F. Liu, Y. Kitabatake, S. Hayase, T. Toyoda, K. Yoshino, T. Minemoto, K. Katayama, and Q. Shen, Nano Energy 53, 17 (2018).

${ }^{23}$ R. Singh, S. Ghosh, A. S. Subbiah, N. Mahuli, and S. K. Sarkar, Sol. Energy Mater. Sol. Cells 205, 110289 (2020).

${ }^{24}$ K. Domanski, B. Roose, T. Matsui, M. Saliba, S. H. Turren-Cruz, J. P. CorreaBaena, C. R. Carmona, G. Richardson, J. M. Foster, F. De Angelis, J. M. Ball, A. Petrozza, N. Mine, M. K. Nazeeruddin, W. Tress, M. Grätzel, U. Steiner, A. Hagfeldt, and A. Abate, Energy Environ. Sci. 10, 604 (2017).

${ }^{25}$ W. Tress and O. Inganäs, Sol. Energy Mater. Sol. Cells 117, 599 (2013).

${ }^{26}$ W. Tress, N. Marinova, T. Moehl, S. M. Zakeeruddin, M. K. Nazeeruddin, and M. Grätzel, Energy Environ. Sci. 8, 995 (2015).

${ }^{27}$ F. Wu, R. Pathak, K. Chen, G. Wang, B. Bahrami, W.-H. Zhang, and Q. Qiao, ACS Energy Lett. 3, 2457 (2018).

${ }^{28}$ L. Bertoluzzi, R. S. Sanchez, L. Liu, J.-W. Lee, E. Mas-Marza, H. Han, N.-G. Park, I. Mora-Sero, and J. Bisquert, Energy Environ. Sci. 8, 910 (2015).

${ }^{29}$ O. Almora, I. Zarazua, E. Mas-Marza, I. Mora-Sero, J. Bisquert, and G. GarciaBelmonte, J. Phys. Chem. Lett. 6, 1645 (2015).

${ }^{30}$ V. K. Sangwan, M. Zhu, S. Clark, K. A. Luck, T. J. Marks, M. G. Kanatzidis, and M. C. Hersam, ACS Appl. Mater. Interfaces 11, 14166 (2019).

${ }^{31}$ F. Galatopoulos, A. Savva, I. T. Papadas, and S. A. Choulis, APL Mater. 5, 076102 (2017).

${ }^{32}$ O. Almora, C. Aranda, I. Zarazua, A. Guerrero, and G. Garcia-Belmonte, ACS Energy Lett. 1, 209 (2016).

${ }^{33}$ G. Garcia-Belmonte and J. Bisquert, ACS Energy Lett. 1, 683 (2016).

${ }^{34}$ X. Shi, Y. Ding, S. Zhou, B. Zhang, M. Cai, J. Yao, L. Hu, J. Wu, S. Dai, and M. K. Nazeeruddin, Adv. Sci. 6, 1901213 (2019).

${ }^{35}$ L. Zhou, Y. Zuo, T. K. Mallick, and S. Sundaram, Sci. Rep. 9, 8778 (2019).

${ }^{36}$ S.-M. Yoo, S. J. Yoon, J. A. Anta, H. J. Lee, P. P. Boix, and I. Mora-Seró, Joule 3, 2535 (2019).

${ }^{37}$ P. Schulz, E. Edri, S. Kirmayer, G. Hodes, D. Cahen, and A. Kahn, Energy Environ. Sci. 7, 1377 (2014).

${ }^{38} \mathrm{~J}$. Carrillo, A. Guerrero, S. Rahimnejad, O. Almora, I. Zarazua, E. Mas-Marza, J. Bisquert, and G. Garcia-Belmonte, Adv. Energy Mater. 6, 1502246 (2016). 